\title{
UGT2B17 genotype and pharmacokinetic profile of testosterone during substitution therapy
}

\author{
A. Kirstine Bang, Niels Jørgensen, Ewa Rajpert-De Meyts and Anders Juul, \\ Department of Growth \& Reproduction, Copenhagen University Hospital (Rigshospitalet), Denmark
} $\triangle$ anne.kirstine.bang@rh.regionh.dk

Introduction

- Testosterone is mainly excreted in the urine as testosterone glucuronide. Glucuronidation is partly dependent on the UGT2B17 genotype (1) and testosterone glucuronide excretion is lower in men having the UGT2B17 deletion polymorphism (2-4).

- We investigated if UGT2B17 genotypes were associated with serum levels of reproductive hormones in hypogonadal men receiving testosterone substitution.

\section{Material \& Methods}

- 207 hypogonadal men treated with Testosterone undecanoate (TU, Nebido®) retrospectively included, and genotyped for the UGT2B17 deletion polymorphism.

- All men had been given 1000 mg TU per injection at start, at 6 and 18 weeks. Subsequent dosage intervals were individualised.

- Reproductive hormone levels were determined in blood samples taken 2 and 6 weeks after the $1^{\text {st }}$ and $2^{\text {nd }}$ injection as well as prior to the $3^{\text {rd }}$ injection and at controls 2-3 years after initiation of treatment.
Table 1. Primary diagnoses stratified according to the UGT2B17 genotype.

\begin{tabular}{|c|c|c|c|c|}
\hline Diagnoses & $\begin{array}{c}\text { ins/ins } \\
\mathrm{n}=87(42 \%)\end{array}$ & $\begin{array}{c}\text { ins } / \text { del } \\
\mathrm{n}=91(44 \%)\end{array}$ & $\begin{array}{c}\text { del } / \text { del } \\
n=29(14 \%)\end{array}$ & $\begin{array}{c}\text { Total } \\
\mathrm{n}=\mathbf{2 0 7}(100 \%)\end{array}$ \\
\hline Klinefelter Syndrome and 46XX,male & $16(39)$ & $20(48.8)$ & $5(12.2)$ & $41(100)$ \\
\hline Primary testicular disease, exl 47XXY & $46(42.6)$ & $49(45.4)$ & $13(12)$ & $108(100)$ \\
\hline Kallmann Syndrome & $10(45.5)$ & $6(27.3)$ & $6(27.3)$ & $22(100)$ \\
\hline Hypogonadotropic hypog., other reasons & $4(40)$ & $5(50)$ & $1(10)$ & $10(100)$ \\
\hline Irradiation induced hypogonadism & $3(50)$ & $3(50)$ & 0 & $6(100)$ \\
\hline Others* & $8(40)$ & $8(40)$ & $4(20)$ & $20(100)$ \\
\hline
\end{tabular}

${ }^{*}$ Others: Injury/torsio/aplasia of the testis $(n=7)$, Fragile $X$ syndrome $(n=1)$, Charge syndrome $(n=1)$, Kennedy syndrome $(n=1)$, Partial Androgen insensitivity syndrome $(n=3)$, Y-microdeletion $(n=1)$, Liver transplant $(n=1)$, Heart transplant $(n=1), \operatorname{HIV}(n=3)$ and Hypogonadism induced by opiods $(\mathrm{n}=1)$

\section{Results}

- The overall frequency of the UGT2B17 genotypes was ins/ins: $42.0 \%$, ins/del: $44.0 \%$ and del/del: $14.0 \%$ (Table 1 ).

- Testosterone levels did not differ between the 3 genotypes 18 weeks after initiation of TU treatment (Figure $1 \mathrm{~A})$.

- LH levels were significantly lower in the del/del group after 18 weeks of TU treatment (Figure 1B). At the same time Estradiol levels tended to be higher in the same group compared to the two other genotype-groups (ins/del and ins/ins) though non-significantly (Figure 1C). SHBG, Free testosterone, total cholesterol or haemoglobin levels did not differ between groups.

- The del/del group had a significantly lower delta testosterone value (8 weeks to 18 weeks) $(p=0.044)$.

- At follow-up 2-3 years after initiation of the treatment, all patients had individual treatment regimes, which were not associated to their UGT2B17 genotype.
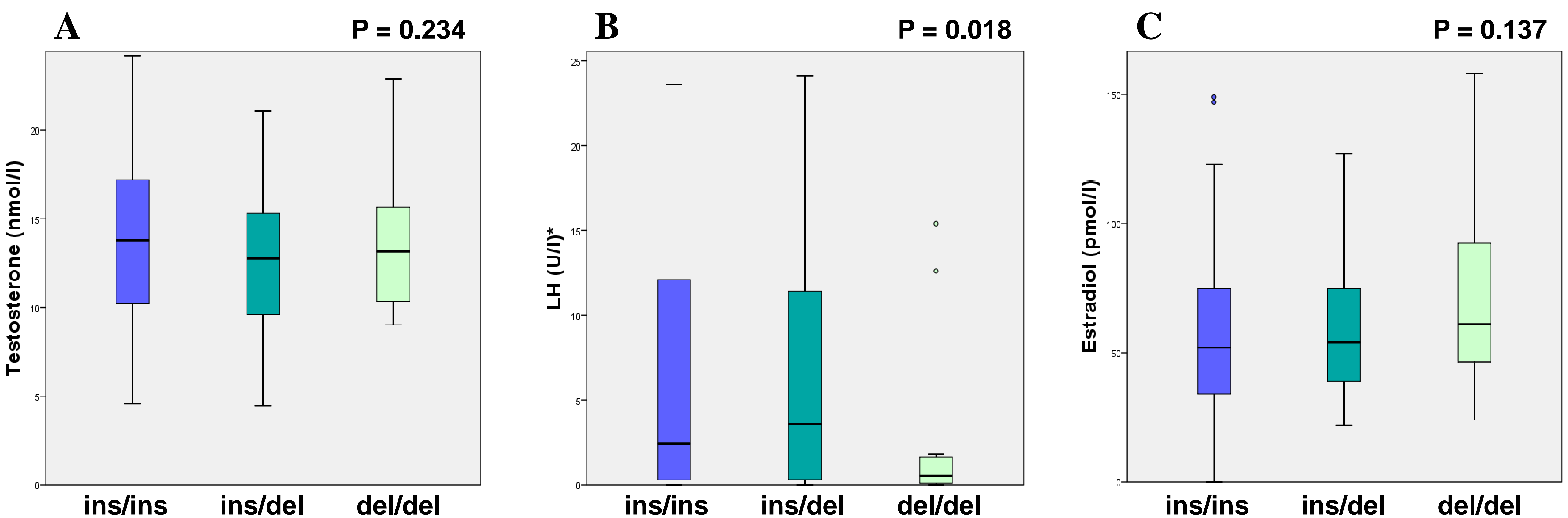

Figure 1.

- Hormone levels in blood samples taken prior to the $3^{\text {rd }}$ injection and stratified according to UGT2B17 genotypes.

-P-values are based on regression analysis adjusted for confounders $(n=166)$.

* LH levels from patients with Hypogonadotropic hypogonadism were not included.

\section{Conclusion}

Serum testosterone levels at 18 weeks did not depend on UGT2B17 genotype in hypogonadal men given standard treatment with TU However the del/del group had a slower fall in testosterone levels in the period between the two last injections as well as lower levels of $\mathrm{LH}$ at 18 weeks. This could indicate a lower testosterone excretion rate, and maybe a possible need of a reduction in testosterone dosage. These findings remain to be investigated further. 